\title{
Characteristic measurements of silicon dioxide aerogel plasmas generated in a Planckian radiation environment
}

Quan-Li Dong, Shou-Jun Wang, Yu-Tong Li, Yi Zhang, Jing Zhao, Hui-Gang Wei, Jian-Rong Shi, Gang Zhao, JiYan Zhang, Yu-Qiu Gu, Yong-Kun Ding, Tian-Shu Wen, Wen-Hai Zhang, Xin Hu, Shen-Ye Liu, Lin Zhang, YongJian Tang, Bao-Han Zhang, Zhi-Jian Zheng, Hiroaki Nishimura, Shinsuke Fujioka, Fei-Lu Wang, Hideaki Takabe, and Jie Zhang

Citation: Physics of Plasmas 17, 012701 (2010); doi: 10.1063/1.3274449

View online: https://doi.org/10.1063/1.3274449

View Table of Contents: http://aip.scitation.org/toc/php/17/1

Published by the American Institute of Physics

\section{Articles you may be interested in}

Opacity measurement of a gold plasma at $\mathrm{T}_{\mathrm{e}}=85 \mathrm{eV}$

Physics of Plasmas 18, 113301 (2011); 10.1063/1.3660407

Calibration of a flat field soft x-ray grating spectrometer for laser produced plasmas

Review of Scientific Instruments 81, 10E319 (2010); 10.1063/1.3495790

Effects of nonequilibrium particle distributions in deuterium-tritium burning

Physics of Plasmas 17, 012707 (2010); 10.1063/1.3276103

Improving a high-efficiency, gated spectrometer for $\mathrm{x}$-ray Thomson scattering experiments at the National Ignition Facility

Review of Scientific Instruments 87, 11E515 (2016); 10.1063/1.4959874

Measurements of the sound velocity of shock-compressed liquid silica to $1100 \mathrm{GPa}$

Journal of Applied Physics 120, 235901 (2016); 10.1063/1.4972338

Enhanced x-ray emissions from Au-Gd mixture targets ablated by a high-power nanosecond laser Journal of Applied Physics 115, 043305 (2014); 10.1063/1.4863143 


\title{
Characteristic measurements of silicon dioxide aerogel plasmas generated in a Planckian radiation environment
}

\author{
Quan-Li Dong, ${ }^{1}$ Shou-Jun Wang, ${ }^{1}$ Yu-Tong Li,,${ }^{1}$ Yi Zhang, ${ }^{1}$ Jing Zhao, ${ }^{1}$ Hui-Gang Wei, ${ }^{2}$ \\ Jian-Rong Shi, ${ }^{2}$ Gang Zhao, ${ }^{2}$ Ji-Yan Zhang, ${ }^{3}$ Yu-Qiu Gu, ${ }^{3}$ Yong-Kun Ding, ${ }^{3}$ \\ Tian-Shu Wen, ${ }^{3}$ Wen-Hai Zhang, ${ }^{3}$ Xin Hu, ${ }^{3}$ Shen-Ye Liu, ${ }^{3}$ Lin Zhang, ${ }^{3}$ Yong-Jian Tang, ${ }^{3}$ \\ Bao-Han Zhang, ${ }^{3}$ Zhi-Jian Zheng, ${ }^{3}$ Hiroaki Nishimura, ${ }^{4}$ Shinsuke Fujioka, \\ Fei-Lu Wang, ${ }^{4}$ Hideaki Takabe, ${ }^{4}$ and Jie Zhang ${ }^{1,5}$ \\ ${ }^{1}$ Beijing National Laboratory of Condensed Matter Physics, Institute of Physics, \\ Chinese Academy of Sciences, Beijing 100190, China \\ ${ }^{2}$ National Astronomical Observatories of China, Chinese Academy of Sciences, Beijing 100012, China \\ ${ }^{3}$ Research Center for Laser Fusion, China Academy of Engineering Physics, Mianyang 621900, China \\ ${ }^{4}$ Institute of Laser Engineering, Osaka University, 2-6 Yamada-Oka, Suita, Osaka 565-0871, Japan \\ ${ }^{5}$ Department of Physics, Shanghai Jiaotong University, Shanghai 200240, China
}

(Received 16 March 2009; accepted 23 November 2009; published online 6 January 2010)

\begin{abstract}
The temporally and spatially resolved characteristics of silicon dioxide aerogel plasmas were studied using $\mathrm{x}$-ray spectroscopy. The plasma was generated in the near-Planckian radiation environment within gold hohlraum targets irradiated by laser pulses with a total energy of $2.4 \mathrm{~kJ}$ in $1 \mathrm{~ns}$. The contributions of silicon ions at different charge states to the specific components of the measured absorption spectra were also investigated. It was found that each main feature in the absorption spectra of the measured silicon dioxide aerogel plasmas was contributed by two neighboring silicon ionic species. (c) 2010 American Institute of Physics. [doi:10.1063/1.3274449]
\end{abstract}

\section{INTRODUCTION}

Experimental and theoretical determination of plasma properties has long been of interests in astrophysics ${ }^{1,2}$ and inertial confinement fusion researches. ${ }^{3}$ The plasma opacity, for instance, which determines the energy transport in plasmas, has been studied extensively in laser-plasma experiments in the past two decades. In those experiments, low and medium- $\mathrm{Z}$ materials are the most early selected as study objects. This is because the K-shell structures and the absorption spectra of low- $\mathrm{Z}$ elements are relatively simple. Aluminum is one of the sample materials usually applied in the opacity model benchmark experiments. ${ }^{4-12}$ In some experiments, aluminum atoms were also buried in the sample for characterization of plasma conditions. Winhart et al. ${ }^{9}$ and Merdji et al. ${ }^{10}$ performed absorption spectra measurements of aluminum plasmas at temperatures $T_{e}$ between 20 and $30 \mathrm{eV}$ and densities $N_{e} \sim 2 \times 10^{20} \mathrm{~cm}^{-3}$, but in different photon energy ranges of 70-280 eV (L-shell) and 1400-1650 eV (K-shell), respectively. Audebert et al. ${ }^{13}$ measured the timeresolved absorption spectra of 300 fs laser-produced, strongly coupled aluminum plasmas of $T_{e} \sim 50 \mathrm{eV}$ and electron density near $5 \times 10^{22} \mathrm{~cm}^{-3}$. Simulations with collisional-radiative atomic physics model included in hydrodynamic code reproduced fairly well the measured charge state distribution as a function of time.

Radiative opacities of medium- $Z$ elements have been largely studied for their key roles in radiation diffusion models of the Sun and other stars, although they represent only a small fraction of the total mass of stars. ${ }^{14}$ Inclusion of transitions with $\Delta n=0$ in the iron opacity calculation, for example, was demonstrated to be the right way in resolving a number of outstanding problems in astrophysics. ${ }^{1,2}$ Da Silva et al. ${ }^{15}$ Springer et al., ${ }^{16}$ and Winhart et al. ${ }^{8}$ measured iron plasma opacities in the photon energy range between 50 and $300 \mathrm{eV}$ due to $\mathrm{M}$-shell transitions under plasma conditions of $T_{e}<60 \mathrm{eV}$ and densities around $10^{20} \mathrm{~cm}^{-3}$. Bailey et al. ${ }^{17}$ performed iron plasma opacity measurements over the photon energy range between 800 and $1800 \mathrm{eV}$, associated with the bound-bound transitions involving the iron L-shell, which is important for solar interior radiation transport. Their plasma was determined to have $T_{e} \sim 156 \pm 6 \mathrm{eV}$ and electron density near $6.9 \pm 1.7 \times 10^{21} \mathrm{~cm}^{-3}$. Foord et al. ${ }^{18,19}$ measured the absorption spectra of iron L-shell in plasmas with $T_{e} \sim 150 \mathrm{eV}$ and density as low as $10^{-4} \mathrm{~g} \mathrm{~cm}^{-3}$ in the two-body recombination regime, and made the first comparison between the measured charge state distributions and that from x-ray photoionization models.

Properties of high- $Z$ element plasmas are also attracting many interests for their wide applications in high power devices such as hohlraums and Z-pinches. ${ }^{20-22}$ Foord et al. studied the emission spectra of laser-produced Au plasmas in order to understand nonlocal thermodynamic equilibrium (non-LTE) processes in the complex M- and N-shell atomic systems. Heeter et al. ${ }^{22}$ performed benchmark measurements of the ionization balance of well-characterized gold plasmas with and without external radiation fields at electron densities near $10^{21} \mathrm{~cm}^{-3}$ and temperatures between 800 and $2400 \mathrm{eV}$. This is the extension work of the benchmark NOVA data for plasmas with $T_{e}$ between 2 and $2.6 \mathrm{keV}$ and densities near $10^{21} \mathrm{~cm}^{-3} .{ }^{21}$ Besides hohlraum device and Z-pinch facility, the Electron Beam Ion Trap (EBIT) facility was also used in benchmarks of the charge state distribution of highly ionized gold plasmas in coronal equilibrium. ${ }^{23}$

With the high-energy transmission grating spectrometer on the Chandra's astrophysical observatory, the spectra with high spectral resolution were observed for the active galactic 


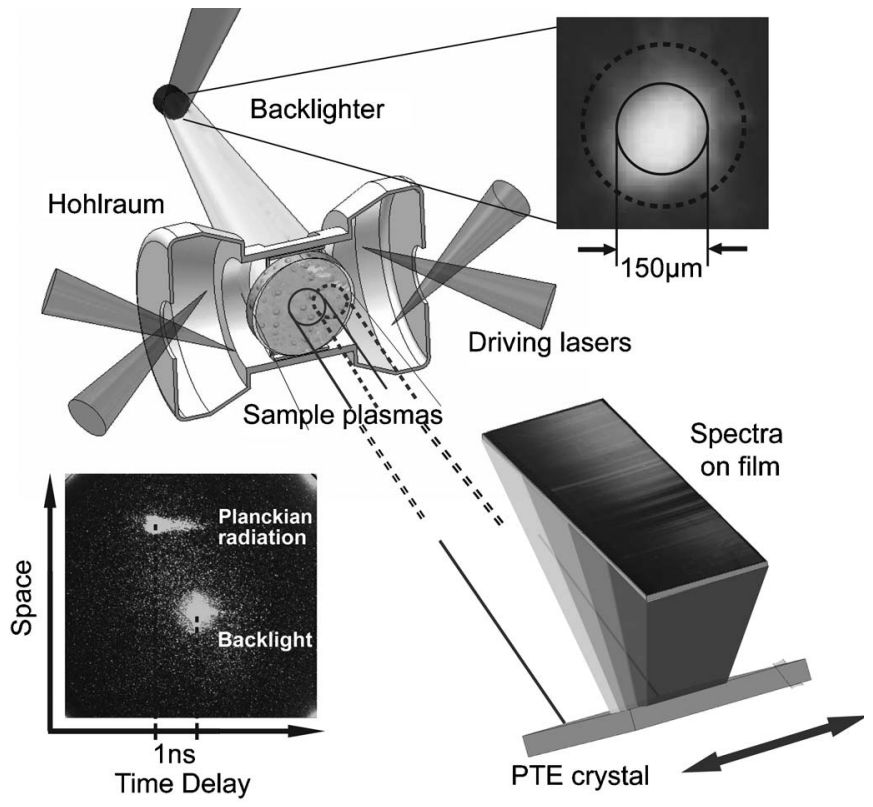

FIG. 1. Graph of basic experimental setup. One typical image of the backlighter is shown at the top right. Besides the uniform backlighting area in the circled center, a ring of weak x-ray emission also exists, indicating the expansion of the plasma. A typical spatial and temporal image of the backlighter and the Planckian radiation is also shown at the down left.

nuclei. $^{24,25}$ Those absorption spectra show evidence of numerous absorption lines from the full range of charge states of silicon and iron. Iron plays important roles in modeling of stars, and has been studied extensively as shown above. Silicon is one of the main opacity sources in the atmospheres of cool stars. ${ }^{26}$ Its line emission can be used as diagnosis of the solar flare. ${ }^{27,28}$ In this paper, we report the study of properties of the silicon dioxide $\left(\mathrm{SiO}_{2}\right)$ foam plasmas at LTE generated by an uniform near-Planckian radiation in hohlraum targets driven with intense laser pulses. The $\mathrm{SiO}_{2}$ plasma absorption spectra were measured in the photon energy range from 1700 to $1900 \mathrm{eV}$. The absorption features in the spectra are due to transitions mainly associated with levels of silicon K-shell. By comparing the measured spectra and the calculated ones with the detailed-term-accounting (DTA) model in assumption of LTE, the plasma temperatures, the areal and the volume densities were determined, respectively. The temporal evolutions of those physical variables were also investigated.

\section{EXPERIMENTS}

The absorption experiments were performed on the Shenguang II Laser facility in Shanghai. Figure 1 gives the scheme of the basic experimental setup, showing the positions of backlighter, sample, crystal, and x-ray film with absorption spectra recorded. Two gold cylindrical hohlraum targets with diameter of $1800 \mu \mathrm{m}$ and length of $1000 \mu \mathrm{m}$ were aligned on their axes and stuck together with a separation distance of $1000 \mu \mathrm{m}$ left for placement of the $\mathrm{SiO}_{2}$ aerogel sample disk tamped by $\mathrm{CH}$ layer on both surfaces. One hole of $\phi 500 \mu \mathrm{m}$ was opened on both outside ends of the organized hohlraum, each allowing entrance of four $0.35 \mu \mathrm{m}$ laser pulses irradiating the inside wall of the
$\mathrm{Au}$ hohlraum, and producing a clean near-Planckian radiation environment. Here by using "clean," we mean that no $\mathrm{Au}$ plasma down from hohlraum wall flowed into the radiation-plasma interaction region. With a total energy of $2.4 \mathrm{~kJ}$ launched in $1 \mathrm{~ns}$, the Planckian radiation was generated with the peak temperature $T_{\text {rmax }} \sim 100 \mathrm{eV}$ as measured with a soft $\mathrm{x}$-ray transmission grating spectrometer. ${ }^{12}$ The $\mathrm{SiO}_{2}$ foam sample disks had diameter of $800 \mu \mathrm{m}$, thickness of $250 \mu \mathrm{m}$, and density of $45 \mathrm{mg} / \mathrm{cm}^{3}$.

The backlight was generated by one $0.7 \mu \mathrm{m}$ laser beam irradiating a gold fiber with cross section of $150 \mu \mathrm{m}$ in diameter, which was $2500 \mu \mathrm{m}$ perpendicularly behind the sample disk. The laser beam had a total energy of $200 \mathrm{~J}$ in 150 ps. Its focus diameter was deliberately adjusted to $\sim 300 \mu \mathrm{m}$, larger than the fiber diameter, helping to produce a uniform backlighting source, which was monitored by an $\mathrm{x}$-ray pinhole camera with magnification ratio of 7 . One typical image of the backlighter is shown at the top of Fig. 1. Besides one uniform area in the center, the image also shows the expansion of the backlighting plasma by a weak ring of $\mathrm{x}$-ray emission on the edge. At each shot, the backlight duration was measured by a streak camera to be around 200 ps, which determined the comparable time resolution in the present experiments.

The primary diagnostics was a flat pentaerythritol tetrakis (hydroxymethy) (PET) methane $\mathrm{C}\left(\mathrm{CH}_{2} \mathrm{OH}\right)_{4}$ crystal spectrometer, which was aligned along the central line that is through the centers of the backlighter and the sample, viewing the sample face-on, dispersing the transmitted backlight and recording on the $\mathrm{x}$-ray film the spectral information between 1700 and $1900 \mathrm{eV}$. The distance between the x-ray recording film and the sample was $\sim 250 \mathrm{~mm}$. The experimental arrangement provided a spectral resolution power of $E / \Delta E \sim 2000$ at $1850 \mathrm{eV}$. The spatial resolution of the PET crystal spectrometer was also limited. Only the transmission of the backlight through the sample region of $250 \mu \mathrm{m}$ was recorded on a $25 \mathrm{~mm}$ wide $\mathrm{x}$-ray film. It was possible to align the crystal hundreds of micrometers away from the central line, allowing measurements of the backlight transmission through the edge part of the sample plasma disk.

We measured the spectra of the backlight transmission through the sample plasma at different times by changing delay periods between the Planckian radiation and the backlight. The time delay was measured by two methods, one of which was to measure the delay between the half height point on the tail of the Planckian-radiation-generating laser pulse and that of the backlighter-generating laser pulse. The other method was to measure directly the time delay between Planckian radiation and the backlight by an x-ray streak camera. A typical spatial and temporal image of the two x-ray sources is also shown at the bottom of Fig. 1. We also measured the self-emission spectra of the backlight and the sample plasma in separate shots. All the data, recorded on the film, were scanned to convert from the film exposure to a computer compatible format, after which, the film fog was numerically corrected. 


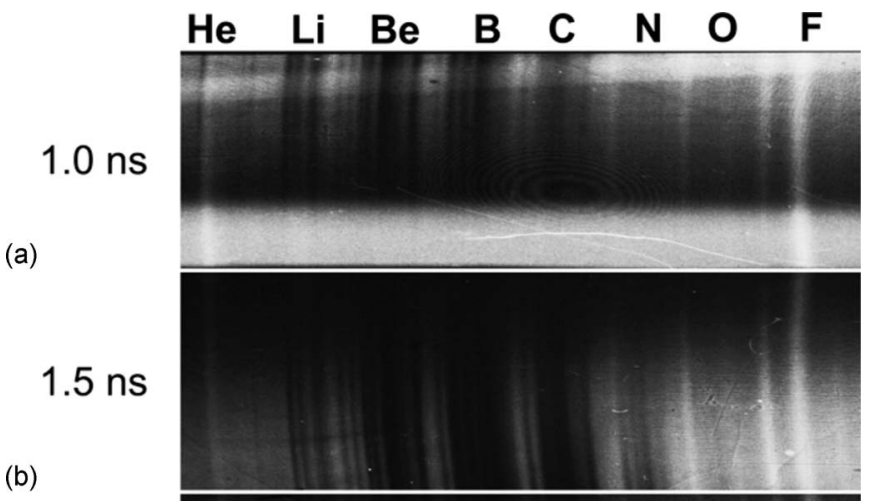

(b)

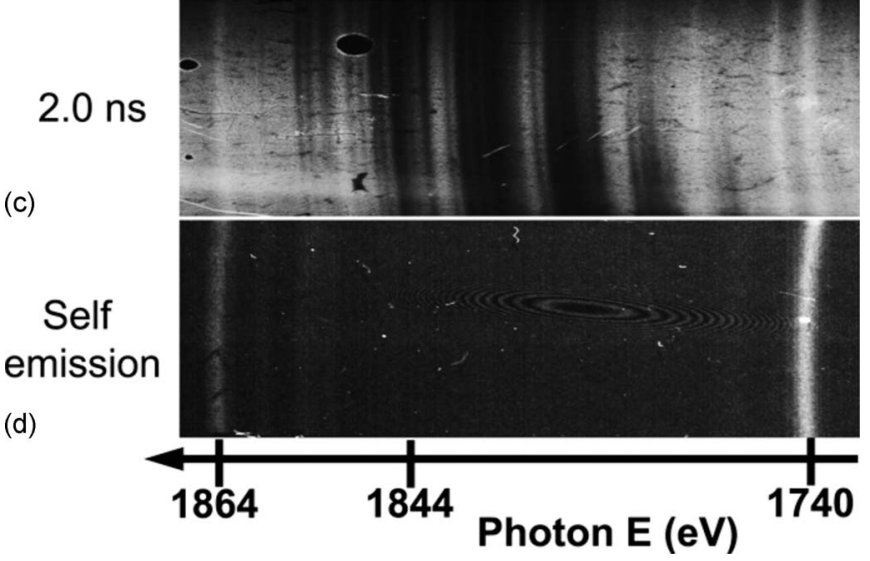

FIG. 2. Film recorded absorption spectra at three delay times of (a) $1.0 \mathrm{~ns}$, (b) $1.5 \mathrm{~ns}$, and (c) $2.0 \mathrm{~ns}$. (a) and (b) were measured when the PET crystal was aligned along the central line of the backlighter and the sample, and (c) was measured for the sample plasmas centered around the position at $150 \pm 25 \mu \mathrm{m}$ away from the central line. (d) is the time-integrated selfemission spectra of the sample plasma. Emission lines from the fluorinlike and heliumlike silicon ions dominate the whole spectra.

\section{X-RAY ABSORPTION SPECTRUM MEASUREMENTS AND DATA ANALYSIS}

Figure 2 shows the film-recorded absorption spectra measured at three different time delays and two different positions. Figures 2(a) and 2(b) show, respectively, the backlight transmission spectra at time delays of 1.0 and $1.5 \mathrm{~ns}$ when the PET crystal was aligned along the central line. Figure 2(c) shows the measured spectra at time delay of $2.0 \mathrm{~ns}$ when the crystal was aligned in such a way that the $\mathrm{x}$-ray film recorded the backlight transmission through sample plasma $150 \pm 25 \mu \mathrm{m}$ away from the central part. All the graphs are arranged with the spectra dispersed in the horizontal direction and the spatial resolution in the vertical. In all three spectra, absorption features by ions from lithiumlike to nitrogenlike are clearly presented. It is noticed that in Figs. 2(a) and 2(b), the continuum is not uniform in the spatial resolution direction; however, the normalized transmission spectra are identical in spectral components for different vertical positions after the division of the continuum spectra. We will show below that the sample plasma at the central part has good uniformity in diameters of about $250 \mu \mathrm{m}$ before time delay of $1.5 \mathrm{~ns}$. Figure 2(c) shows different features of the spectral components in different vertical positions, indicating the nonuniformity of the sample plasma characteristics at the edge. It is noted that the bottom part of Fig. 2(a) was overexposed a little, and the absorption features were blurred. The randomly curved bright line in Fig. 2(a) and the black dots in Fig. 2(c) were defects of the film. In Fig. 2(d), the time-integrated self-emission spectra of the $\mathrm{SiO}_{2}$ sample plasma are given, showing the dominating feature lines of heliumlike and fluorinlike silicon ions.

\section{A. Description of atomic model used}

We analyze the experimental spectra by comparing them with the calculated ones with the DTA model, for the DTA calculation has been shown to reproduce the experimental absorption spectra with excellent agreement. ${ }^{29}$ In our calculation, the ionization balance is provided with Saha equations and the energy level population from the Boltzmann distribution, assuming the plasma in LTE. The Debye-Huckel model is used to calculate the ionization potential depression. The necessary atomic data are computed by the flexible atomic code (FAC). ${ }^{30}$ In the calculations, we have taken into account the four kinds of line profile broadening mechanisms. They are the natural width, the autoionization resonance width, the Doppler broadening width, and the Stark broadening width, among which, the first two can be calculated by FAC. The natural width, $50 \mathrm{meV}$ for instance, is usually smaller than those due to other broadening mechanisms. The autoionization resonance broadening width due to the mixing between electronic configurations decreases as the ion charge increases. The calculation produces autoionization resonance width of oxygenlike ions from 0.1 to $0.25 \mathrm{eV}$ with the typical value of $0.2 \mathrm{eV}$, while that of berylliumlike ions from 0 to $0.08 \mathrm{eV}$ with the typical value of $0.04 \mathrm{eV}$, comparable to the natural width. The Doppler broadened line has a Gaussian profile with a width $\Gamma_{d}$ as

$$
\Gamma_{d}=h \nu \frac{2}{c} \sqrt{2 \ln 2 \frac{k_{B} T_{e}}{m_{i}}},
$$

where $c$ is the light speed in vacuum, $k_{B}$ the Boltzmann constant, and $m_{i}$ the mass of the ions. All above physical variables are in their corresponding units of international system (SI) except the photon energy $h \nu$ in $\mathrm{eV}$. For the sample plasma of $T_{e} \sim 50 \mathrm{eV}$, the Doppler broadening width is around $0.17-0.19 \mathrm{eV}$ in the photon energy range between 1700 and $1900 \mathrm{eV}$. For the Stark broadening mechanism, the width $\Gamma_{s}$ of its Lorentzian line profile is calculated by using the simplified semiempirical method instead of the timeconsuming quantum mechanical calculation,

$$
\begin{aligned}
\Gamma_{s}= & N_{e} \frac{4 \pi}{3} \frac{\hbar^{3}}{m^{2} c} \sqrt{\frac{2 m}{\pi k_{B} T}} \frac{\pi}{\sqrt{3}}\left(0.9-\frac{1.1}{z}\right) \\
& \times \sum_{j=i, f}\left(\frac{3 n_{j}}{2 z}\right)\left(n_{j}^{2}-l_{j}^{2}-l_{j}-1\right),
\end{aligned}
$$

where $n_{i}\left(l_{i}\right)$ and $n_{j}\left(l_{j}\right)$ are the effective principal (the orbital angular momentum) quantum numbers of the lower and the upper energy levels of the transition within ions charged of $z$. The absorption line then has a Voigt profile as the convolution of Gaussian and Lorentzian profiles. 


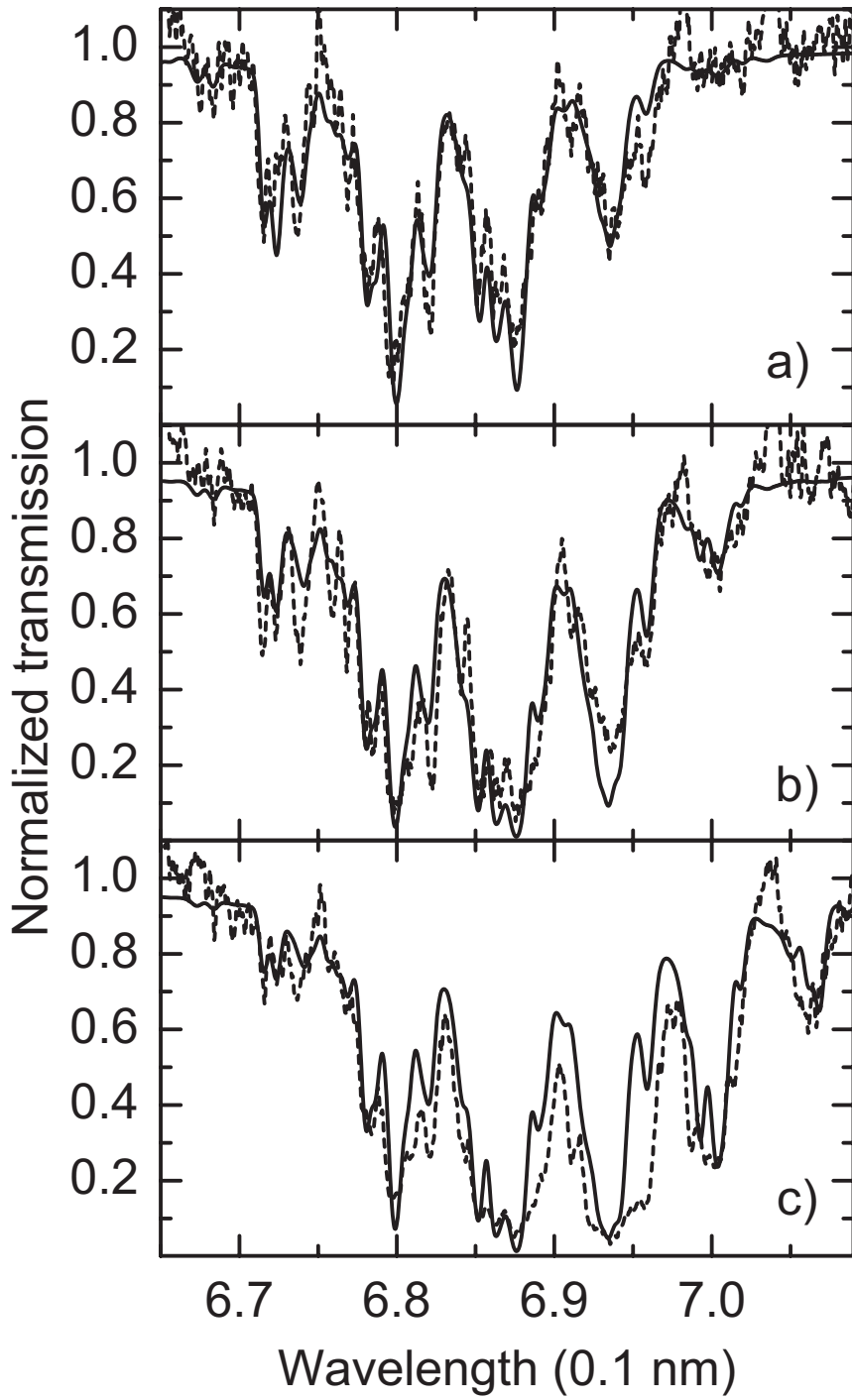

FIG. 3. Comparisons between the measured absorption spectra of plasmas at the sample central region (dashed lines) and the calculation results (solid lines) at delay times of $1.0,1.5$, and $2.0 \mathrm{~ns}$, respectively.

In the experiments, the measured absorption line appears as the convolution of the theoretical profile with the instrumental broadening profile given by

$$
\frac{I_{\text {exp }}(h \nu)}{I_{\text {back }}}=\int_{-\infty}^{+\infty} \frac{I_{\text {th }}\left(h \nu^{\prime}\right)}{I_{\text {back }}} g\left(h \nu-h \nu^{\prime}\right) d\left(h \nu^{\prime}\right),
$$

where

$$
g\left(h \nu^{\prime}\right)=\operatorname{rect}\left(\frac{h \nu^{\prime}}{h \Delta \nu_{\text {ins }}}\right)= \begin{cases}1 / h \Delta \nu_{\text {ins }}, & \left|h \nu^{\prime}\right| \leq h \Delta \nu_{\text {ins }} / 2 \\ 0, & \text { others }\end{cases}
$$

represents the uniform $\mathrm{x}$-ray backlighter.

\section{B. Absorption spectra analysis}

Figures 3(a)-3(c) show the three transmission spectra through the sample center at 1.0, 1.5, and $2.0 \mathrm{~ns}$, respectively. At the time of $1 \mathrm{~ns}$, the transmission spectra feature fine structures for different charge states. As the time delay increases, the strongest absorption series, such as that of boronlike and carbonlike ions, become saturated and broad-

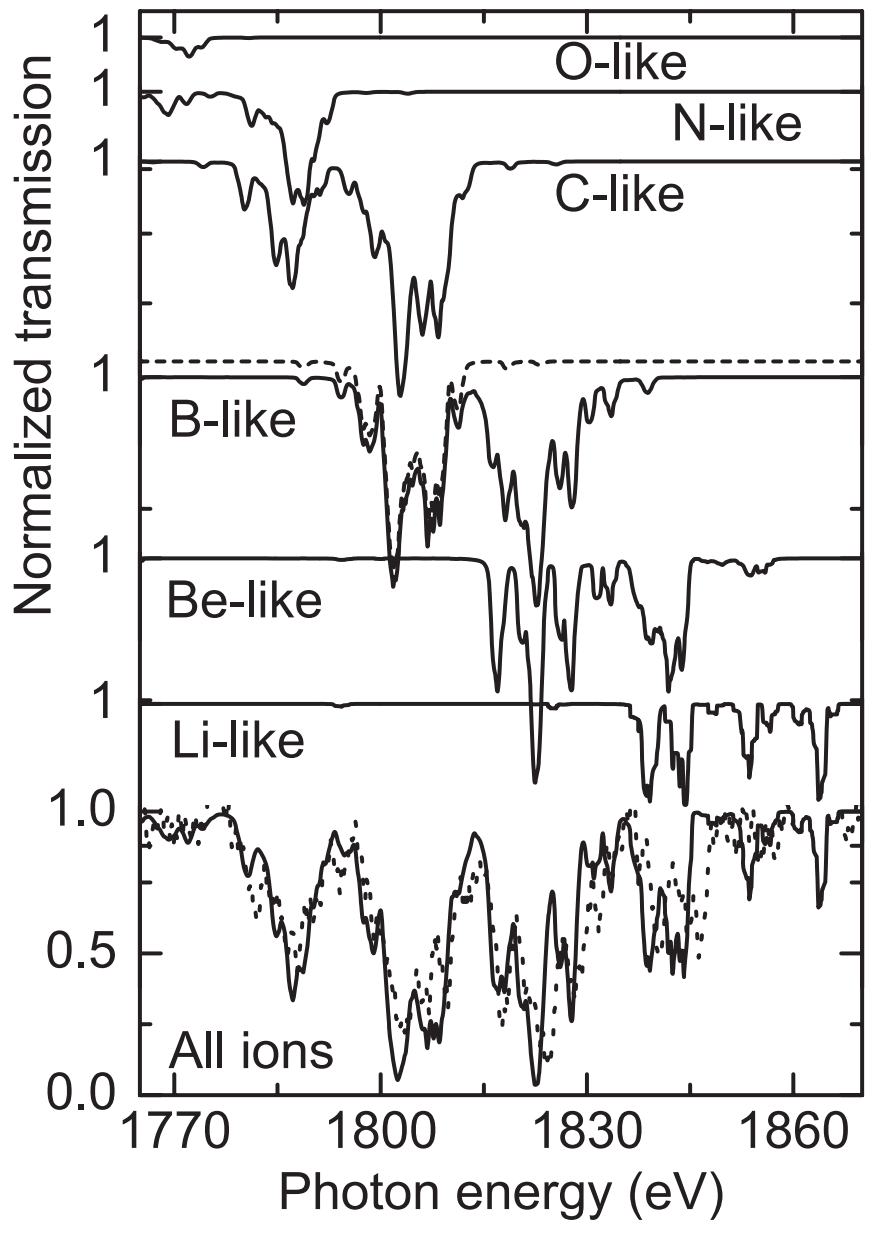

FIG. 4. Contributions of Li-like and O-like silicon ions to the whole absorption spectra at delay time of $1.5 \mathrm{~ns}$. The dashed line is the absorption spectra of B-like silicon ions having electronic configurations with the maximum principal quantum number $n_{\max }=2$. Its intensity was increased by 0.1 manually for the clearness aim. The dotted line is the measured absorption spectra of plasmas in the central sample region.

ened, and the line structures are blurred. The calculated spectra are also plotted and compared with the experimental ones. At delay times of 1.0 and $1.5 \mathrm{~ns}$, the calculations reproduce the experimental spectra with a good agreement under the assumption that there exists one group of sample plasmas at LTE. For the absorption spectra at $2.0 \mathrm{~ns}$, however, there exist simultaneously strong oxygenlike ion features and strong lithiumlike ion features. We use two groups of LTE plasmas with different electron temperatures and densities to reproduce the experimental spectra.

We study the detailed contributions of each charge state to the measured absorption spectra through the central part of the sample plasma at delay time of $1.5 \mathrm{~ns}$ [Fig. 2(b)]. The absorption spectra of each kind of ions are plotted in Fig. 4 when the calculation reproduces the experimental results with good agreements. For boronlike, carbonlike, and nitrogenlike ions, the spectra feature two absorption series, among which the lower energy series are mainly from transitions between energy levels of the electronic configurations with the maximum principal quantum number $n_{\max }=2$, while the other absorption series at higher energy are due to transitions involving electronic configurations with $n_{\max } \geq 3$. As 
an example, the dashed line in Fig. 4 shows the absorption spectra by B-like ions in electronic configurations with $n_{\max }=2$. It is also found that the lower energy series of ions $\mathrm{Si}^{\mathrm{N+}}$ locate at the same photon energy range as the higher energy series of ions $\mathrm{Si}^{(\mathrm{N}-1)+}$. This is because the corresponding absorption series of the two neighboring ions have similar transition behaviors involving the inner shells of electronic configurations. For example, B-like silicon ion has the ground state electronic configuration of $1 s^{2} 2 s^{2} 2 p^{1}$. Its lower energy absorption series are mainly due to the inner shell electron excitation from levels belonging to configurations of $1 s^{2} 2 s^{2} 2 p^{1}, 1 s^{2} 2 s^{1} 2 p^{2}$, and $1 s^{2} 2 p^{3}$. The neighboring C-like silicon ion has the ground state electronic configuration of $1 s^{2} 2 s^{2} 2 p^{2}$. Its higher energy absorption series, however, are mainly due to the inner shell electron excitation from levels of $1 s^{2} 2 s^{2} 2 p^{1} n l, 1 s^{2} 2 s^{1} 2 p^{2} n l$, and $1 s^{2} 2 p^{3} n l$, where $n \geq 3$ and $l=0 \sim n-1$. The state of the most outside electrons usually changes along with the inner shell electron excitation processes as the system parity conservation requires. But such changes introduce minus effects on transitions between levels involving inner shells. For Li- and Be-like silicon ions, the absorption spectra present separate line features. This is because ions with fewer electrons have simple electronic configurations and energy level distributions, and the gaps between levels are usually larger. For the Li-like absorption spectra, the first line from the left in Fig. 4 is due to transitions between energy levels belonging to configurations of $1 s^{2} 2 p^{1}$ and $1 s^{1} 2 p^{2}$. The excitations from the ground electronic configuration $1 s^{2} 2 s^{1}$ to $1 s^{1} 2 s^{1} 2 p^{1}$ contribute much to the second line from the left. Other contributions to the second line are from some transitions involving $1 s^{2} 2 p^{1}$ and $1 s^{1} 2 p^{2}$. The third and the fourth lines are due to the transitions of $1 s^{2} n l$ to $1 s^{1} 2 s^{1} n^{\prime} l^{\prime}$ or $1 s^{1} 2 p^{1} n^{\prime} l^{\prime}$, with $n=3,4$ and $n^{\prime}>n+1$, respectively. It is clear that the absorption series of the charge state $\mathrm{Si}^{\mathrm{N}}$ are usually overlapping with that of ions $\mathrm{Si}^{\mathrm{N}-1}$ except Li-like silicon ions because for He-like ions, the emissions dominate the absorptions, as shown in Fig. 2.

It is noted that some detailed structures are not reproduced well in Fig. 3. The absorption lines measured in experiments around $6.83 \AA$ or $1816.7 \mathrm{eV}$ are deeper than the calculation results with LTE assumption. This absorption series is due to excitations of energy levels of the ground electronic configurations $1 s^{2} 2 s^{2}$ to that of $1 s^{1} 2 s^{2} 2 p^{1}$, and $1 s^{2} 2 p^{2}$ to $1 s^{1} 2 p^{3}$ of Be-like silicon ions. Transitions involving electronic configurations of B-like silicon ions at excited states $1 s^{2} 2 p^{2} n l$ to $1 s^{1} 2 p^{3} n^{\prime} l^{\prime}$ also contribute to this absorption series. It is the same situation for the absorption series around $1780 \mathrm{eV}$, to which transitions between energy levels of the ground electronic configurations of C-like silicon ions and $\mathrm{N}$-like silicon ions contribute. The mismatch between experimental data and the calculation results might be attributed to several aspects. First, the LTE assumption for the plasma condition might not represent the real situation. Photons of higher energy involving the $\mathrm{M}$-shell transitions of $\mathrm{Au}$ ions in plasmas of hohlraum wall may cause increased photon excitation and ionization over the LTE approximation. Second, effects leading to this mismatch might include the existence of the gradients of velocity, density, and temperature of plasmas. In the reproduction of the measured absorption spectra,

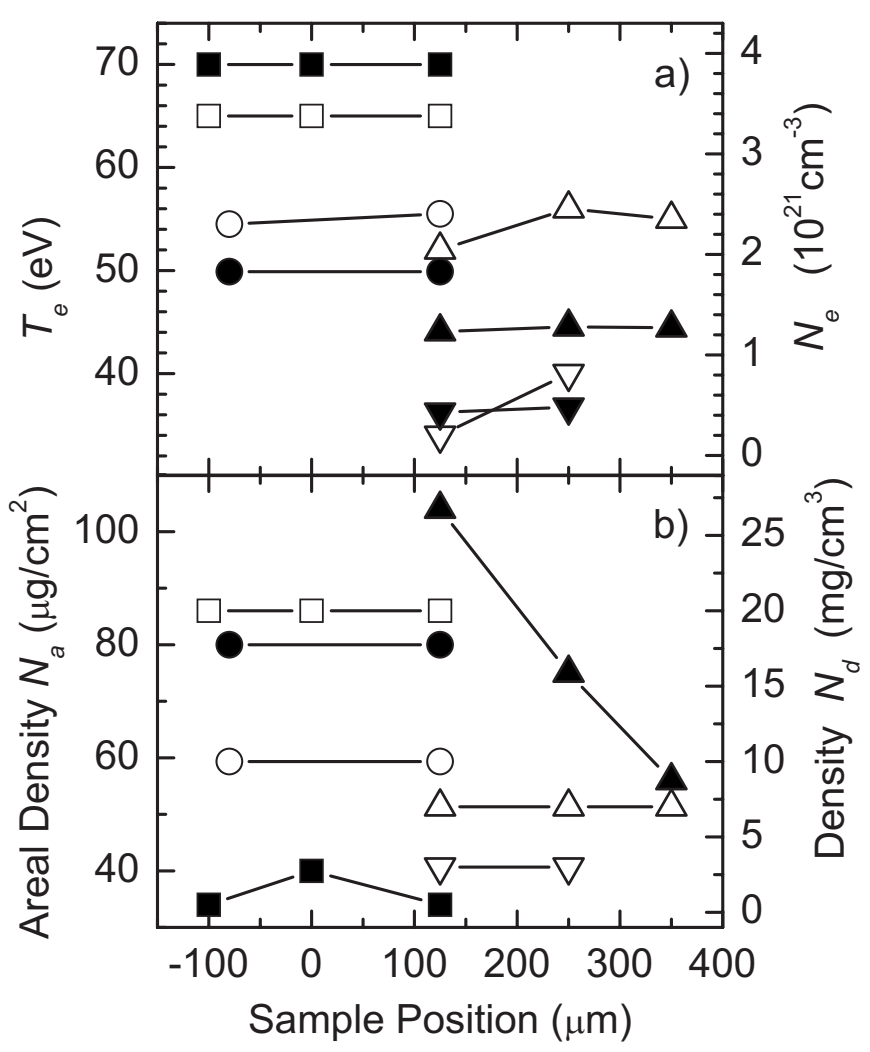

FIG. 5. The temporally and spatially resolved characteristics of silicon dioxide aerogel plasmas. (a) The electron temperature $T_{e}$ (squares and circles are for time delay of 1.0 and $1.5 \mathrm{~ns}$, respectively; up-triangles and downtriangles are for different groups of LTE plasmas at $2.0 \mathrm{~ns}$ ) and the electron density $N_{e}$ (solid squares and solid circles are for delays of 1.0 and $1.5 \mathrm{~ns}$, respectively; solid up-triangles and down-triangles are for different groups of LTE plasmas at the time delay of $2.0 \mathrm{~ns}$ ). (b) The areal density $N_{a}$ (solid squares, solid circles, and solid up-triangles are for delays of 1.0, 1.5, and $2.0 \mathrm{~ns}$, respectively) and the volume density $N_{d}$ (squares and circles are for delays of 1.0 and $1.5 \mathrm{~ns}$, respectively; up-triangles and down-triangles are for different groups of LTE plasmas at the time delay of $2.0 \mathrm{~ns}$ ).

we assumed that the sample plasma consists of two groups of LTE plasmas of different electron temperatures and densities. However, it is expected that the gradients of plasmas should produce a mismatch at the whole energy range, which situation is not our observations. The third effect for the mismatch might be the always-existing errors in the calculated atomic data. The accuracy of atomic data of silicon needs more future detailed works. The saturation behavior of the absorption spectra measured at $2 \mathrm{~ns}$ is due to the areal density increase, as will be shown later in Sec. III C. However, the saturation does not show the characteristic of transmission intensity reaching zero as theory usually predicts, because the absorption spectra of the plasma have been convoluted with the instrumental broadening width when recorded on the x-ray film. ${ }^{4}$

\section{Temporal evolution of plasma properties}

The spatial and temporal evolutions of the sample plasma temperatures $T_{e}$ in $\mathrm{eV}$ and the electron density $N_{e}$ in $\mathrm{cm}^{-3}$ are shown in Fig. 5(a). Figure 5(b) shows the spatial and temporal evolutions of areal densities $N_{a}$ in $\mu \mathrm{g} / \mathrm{cm}^{3}$ and volume densities $N_{d}$ in $\mathrm{mg} / \mathrm{cm}^{3}$. We have $N_{a}=N_{d} \times l$, where 
$l$ in centimeter is the backlight transmission depth in the sample plasma. The sample position zero in Fig. 5 represents the central line of the sample and the backlighter. At delay time of $1.0 \mathrm{~ns}$, the plasma has a good uniformity at the central region. The electron temperature is determined to be $65 \pm 3 \mathrm{eV}$ at three positions, i.e., $-80,0$, and $125 \mu \mathrm{m}$. Here and hereafter, the error in $T_{e}$ determination is added as $\pm 3 \mathrm{eV}$, which is estimated in the way that a change in such value in the electron temperature can cause 0.3 in the average charge and around 10\% changes in the absorption strength by Li-like and $\mathrm{N}$-like silicon ions. The areal density $N_{a} \sim 40 \mu \mathrm{g} / \mathrm{cm}^{2}=20 \mathrm{mg} / \mathrm{cm}^{3} \times 0.0020 \mathrm{~cm}$ for the position 0 , and $34 \mu \mathrm{g} / \mathrm{cm}^{2}=20 \mathrm{mg} / \mathrm{cm}^{3} \times 0.0017 \mathrm{~cm}$ for the other two positions. At delay time of $1.5 \mathrm{~ns}$, the calculations reproduce the measured spectra at a lower temperature $T_{e} \sim 55 \pm 3 \mathrm{eV}$ but a higher $N_{a} \sim 80 \mu \mathrm{g} / \mathrm{cm}^{2}=10 \mathrm{mg} / \mathrm{cm}^{3}$ $\times 0.0080 \mathrm{~cm}$. However, at delay time of $2 \mathrm{~ns}$, this uniformity in the sample center deteriorates. We use two groups of LTE plasmas to reproduce the measured transmission spectra through positions of 125 and $250 \mu \mathrm{m}$. One group of plasma at position $125 \mu \mathrm{m}$ has a temperature $T_{e} \sim 52 \pm 3 \mathrm{eV}$ and $N_{a} \sim 77 \mu \mathrm{g} / \mathrm{cm}^{2}=7 \mathrm{mg} / \mathrm{cm}^{3} \times 0.011 \mathrm{~cm}$, and the other has a temperature $T_{e} \sim 34 \pm 3 \mathrm{eV}$ and $N_{a} \sim 27 \mu \mathrm{g} / \mathrm{cm}^{2}$ $=3 \mathrm{mg} / \mathrm{cm}^{3} \times 0.009 \mathrm{~cm}$. At position $250 \mu \mathrm{m}$, the first group of plasma has $T_{e} \sim 56 \pm 3 \mathrm{eV}$ and $N_{a} \sim 63 \mu \mathrm{g} / \mathrm{cm}^{2}$ $=7 \mathrm{mg} / \mathrm{cm}^{3} \times 0.009 \mathrm{~cm}$. The second group of plasma here has $T_{e} \sim 40 \pm 3 \mathrm{eV}$ and $N_{a} \sim 12 \mu \mathrm{g} / \mathrm{cm}^{2}=3 \mathrm{mg} / \mathrm{cm}^{3}$ $\times 0.004 \mathrm{~cm}$. For the most outside positions at tens of micrometers to the edge of the original sample disk, comparison between calculation and experiment results indicates a uniform plasma region with $T_{e} \sim 55 \pm 3 \mathrm{eV}$ and $N_{a} \sim 56 \mu \mathrm{g} / \mathrm{cm}^{2}=7 \mathrm{mg} / \mathrm{cm}^{3} \times 0.0080 \mathrm{~cm}$. It appears that at later times, the plasma at the sample edge is roughly at LTE and has a higher electron temperature than that in the central region, where, as described below, some thermal energy from the existing plasmas is taken away by electrons transporting into the neutral sample and producing new plasmas through impact ionization. The fact that plasmas at the sample edge have a uniformly distributed temperature might indicate that the temperature of the Planckian radiation then is around $T_{r} \sim 55 \mathrm{eV}$. It is worth to point out that the error in the determined electron density could be larger than that of the electron temperature, since the absorption spectra are not so sensitive to the electron density as compared with the electron temperature, according to our calculations. Detailed studies about the electron density effects on the absorption spectra are needed in further experiments.

In the experiments, two effects may contribute to the evolution characteristics above. The dual temperature might be attributed to the sample plasma expanding out of the hohlraum and cooling down. This implies that the most outside part of the plasma has a low temperature. However, for our plasma with a typical electron temperature $T_{e} \sim 55 \mathrm{eV}$, the expansion speed can be approximated by its ion acoustic wave velocity of $c_{s}=\sqrt{\gamma Z k_{B} T_{e} / m_{i}}=3.88 \times 10^{6} \mathrm{~cm} / \mathrm{s}$, where the adiabatic index $\gamma \sim 1$, and the average ionization stage $Z \sim 8$. Samples with such a speed can expand a distance of $\sim 40 \mu \mathrm{m}$ in $1 \mathrm{~ns}$. This distance is smaller than the hohlraum diameter of $1800 \mu \mathrm{m}$, indicating that the plasma keeps within the Planckian radiation environment during the measurements. Expansion behavior does not contribute much to the nonuniformity of the plasma in the sample central region. Another explanation takes account the plasma generation process. At the early stage of plasma formation, when the Planckian radiation is around $T_{r} \sim 65 \mathrm{eV}$, photons ionize the surface part of the sample into near-LTE plasma with comparable temperature. The transmission backlight spectra measured at this time are those through the left neutral $\mathrm{SiO}_{2}$ aerogel sample tamped by plasmas on both surfaces. After that, the energy transport into the left sample should have taken the forms of radiation as well as thermal conduction by electrons. At later time of $2 \mathrm{~ns}$, electrons and photons continue to ionize the neutral sample into plasma, which consists of the second group with lower temperature and lower density. Plasma expansion does have effects on the absorption spectra by lowering the plasma areal density at the sample disk edge.

\section{SUMMARY}

In summary, we have measured the absorption spectra of plasmas generated by the Planckian radiation within hohlraum targets driven by intense laser pulses. With comparisons between the experimental spectra and the calculation results, the evolution characteristics of the plasma are studied. It is found that the sample with high density in the Planckian radiation environment is gradually ionized into plasmas from the surface into the core by photon ionizations and electron collision ionizations within several nanoseconds. The surface of the sample was photoionized at the early stage into plasmas, but immediately after that, the energy transport has taken the form of the photon flux and the thermal conduction. The plasma is roughly at LTE states during the early measurement period, but non-LTE effect due to the contributions from the photoionization processes is not neglectable as it manifests itself by the mismatch between the experimental spectra and the LTE-assumed calculation results, and by the fact that only with assumption of multigroups of LTE plasmas in the theoretical analysis, is it possible to reproduce the experimental results in a fair agreement.

\section{ACKNOWLEDGMENTS}

This work is jointly supported by the National Natural Science Foundation of China (Grant Nos. 60621063, 60678007, and 10774184), the CAS project of KJCX2-YWT01, and the National Basic Research Program of China (973 Program) under Grant No. 2007CB815101. We also thank all the staff of the National Laboratory on High Power Lasers and Physics.

${ }^{1}$ C. A. Iglesias and F. J. Rogers, Astrophys. J. 371, L73 (1991).

${ }^{2}$ F. J. Rogers and C. A. Iglesias, Science 263, 50 (1994).

${ }^{3}$ J. Lindl, Phys. Plasmas 2, 3933 (1995).

${ }^{4}$ C. Chenais-Popovics, C. Fievet, J. P. Geindre, I. Matsushima, and J. C. Gauthier, Phys. Rev. A 42, 4788 (1990).

${ }^{5}$ T. S. Perry, S. J. Davidson, F. J. D. Serduke, D. R. Bach, C. C. Smith, J. M. Foster, R. J. Doyas, R. A. Ward, C. A. Iglesias, F. J. Rogers, 
J. Abdallah, Jr., R. E. Stewart, J. D. Kilkenny, and R. W. Lee, Phys. Rev. Lett. 67, 3784 (1991).

${ }^{6}$ C. A. Iglesias, M. H. Chen, D. L. McWilliams, J. K. Nash, and F. J. Rogers, J. Quant. Spectrosc. Radiat. Transf. 54, 185 (1995).

${ }^{7}$ T. S. Perry, P. T. Springer, D. F. Fields, D. R. Bach, F. J. D. Serduke, C. A. Iglesias, F. J. Rogers, J. K. Nash, M. H. Chen, B. G. Wilson, W. H. Goldstein, B. Rozsynai, R. A. Ward, J. D. Kilkenny, R. Doyas, L. B. Da Silva, C. A. Back, R. Cauble, S. J. Davidson, J. M. Foster, C. C. Smith, A. Bar-Shalom, and R. W. Lee, Phys. Rev. E 54, 5617 (1996).

${ }^{8}$ G. Winhart, K. Eidmann, C. A. Iglesias, A. Bar-Shalom, E. Mínguez, A. Rickert, and S. J. Rose, J. Quant. Spectrosc. Radiat. Transf. 54, 437 (1995).

${ }^{9}$ G. Winhart, K. Eidmann, C. A. Iglesias, and A. Bar-Shalom, Phys. Rev. E 53, R1332 (1996).

${ }^{10}$ H. Merdji, K. Eidmann, C. Chenais-Popovics, G. Winhart, J. C. Gauthier, A. Mirone, and C. A. Iglesias, J. Quant. Spectrosc. Radiat. Transf. 58, 773 (1997).

${ }^{11}$ D. J. Hoarty, C. D. Bentley, B. J. B. Crowley, S. J. Davidson, S. G. Gales, P. Graham, J. W. O. Harris, C. A. Iglesias, S. F. James, and C. C. Smith, J. Quant. Spectrosc. Radiat. Transf. 99, 283 (2006).

${ }^{12}$ Y. Xu, J. Y. Zhang, J. M. Yang, W. B. Pei, Y. K. Ding, D. X. Lai, G. W. Men, and Z. Luo, Phys. Plasmas 14, 052701 (2007).

${ }^{13}$ P. Audebert, P. Renaudin, S. Bastiani-Ceccotti, J.-P. Geindre, C. Chenais-Popovics, S. Tzortzakis, V. Nagels-Silvert, R. Shepherd, I. Matsushima, S. Gary, F. Girard, O. Peyrusse, and J.-C. Gauthier, Phys. Rev. Lett. 94, 025004 (2005).

${ }^{14}$ G. B. Rybicki and A. P. Lightman, Radiative Processes in Astrophysics (Wiley, New York, 1979).

${ }^{15}$ L. B. Da Silva, B. J. MacGowan, D. R. Kania, B. A. Hammel, C. A. Back, E. Hsieh, R. Doyas, C. A. Iglesias, F. J. Rogers, and R. W. Lee, Phys. Rev. Lett. 69, 438 (1992).

${ }^{16}$ P. T. Springer, D. J. Fields, B. G. Wilson, J. K. Nash, W. H. Goldstein, C. A. Iglesias, F. J. Rogers, J. K. Swenson, M. H. Chen, A. Bar-Shalom, and R. E. Stewart, Phys. Rev. Lett. 69, 3735 (1992).
${ }^{17}$ J. E. Bailey, G. A. Rochau, and C. A. Iglesias, Phys. Rev. Lett. 99, 265002 (2007).

${ }^{18}$ M. E. Foord, R. F. Heeter, P. A. M. van Hoof, R. S. Thoe, J. E. Bailey, M. E. Cuneo, H.-K. Chung, D. A. Liedahl, K. B. Fournier, G. A. Chandler, V. Jonauskas, R. Kisielius, L. P. Mix, C. Ramsbottom, P. T. Springer, F. P. Keenan, S. J. Rose, and W. H. Goldstein, Phys. Rev. Lett. 93, 055002 (2004).

${ }^{19}$ M. E. Foord, R. F. Heeter, H.-K. Chung, P. A. M. van Hoof, J. E. Bailey, M. E. Cuneo, D. A. Liedahl, K. B. Fournier, V. Jonauskas, R. Kisielius, C. Ramsbottom, P. T. Springer, F. P. Keenan, S. J. Rose, and W. H. Goldstein, J. Quant. Spectrosc. Radiat. Transf. 99, 712 (2006).

${ }^{20}$ M. E. Foord, S. H. Glenzer, R. S. Thoe, S. H. Glenzer, R. S. Thoe, K. L. Wong, K. B. Fournier, B. G. Wilson, and P. T. Springer, Phys. Rev. Lett. 85, 992 (2000).

${ }^{21}$ S. H. Glenzer, K. B. Fournier, B. G. Wilson, R. W. Lee, and L. J. Suter, Phys. Rev. Lett. 87, 045002 (2001).

${ }^{22}$ R. F. Heeter, S. B. Hansen, K. B. Fournier, M. E. Foord, D. H. Froula, A. J. Mackinnon, M. J. May, M. B. Schneider, and B. K. F. Young, Phys. Rev. Lett. 99, 195001 (2007).

${ }^{23}$ K. L. Wong, M. J. May, P. Beiersdorfer, K. B. Fournier, B. Wilson, G. V. Brown, P. Springer, P. A. Neill, and C. L. Harris, Phys. Rev. Lett. 90, 235001 (2003).

${ }^{24}$ S. Kaspi, W. N. Brandt, I. M. George, H. Netzer, D. M. Crenshaw, J. R. Gabel, F. W. Hamann, M. E. Kaiser, A. Koratkar, S. B. Kraemer, G. A. Kriss, S. Mathur, R. F. Mushotzky, K. Nandra, B. M. Peterson, J. C. Shields, T. J. Turner, and W. Zheng, Astrophys. J. 574, 643 (2002).

${ }^{25}$ T. Holczer, Astrophys. J. 663, 799 (2007).

${ }^{26}$ S. Wedemeyer, Astron. Astrophys. 373, 998 (2001).

${ }^{27}$ K. J. H. Philips, J. Dubau, J. Sylwester, and B. Sylwester, Astrophys. J. 638, 1154 (2006).

${ }^{28}$ G. Y. Liang, G. Zhao, and J. R. Shi, Astrophys. J. 132, 371 (2006).

${ }^{29}$ J. Abdallah and R. E. H. Clark, J. Appl. Phys. 69, 23 (1991).

${ }^{30}$ M. F. Gu, Astrophys. J. 582, 1241 (2003). 